\title{
Uso de heurísticos en la evaluación de las fuentes históricas en el aula de clase ${ }^{1}$
}

\author{
Virgelina Castellanos Páez², Claudia Patricia Navarro Roldán³ \\ Universidad Pedagógica y Tecnológica de Colombia (Colombia)
}

Recibido: 12/02/2019Ａceptado: 13/12/2019

\begin{abstract}
Resumen
Objetivo. Describir cómo el uso de heurísticos en la evaluación de la evidencia histórica posibilita la construcción del conocimiento sobre la historia de los derechos humanos en una clase de Ciencias Sociales. Método. Estudio cualitativo, con método de análisis del discurso, para analizar cómo la maestra y sus estudiantes de $8^{\circ}$ grado $(n=32)$ usan y evalúan la evidencia histórica. Cinco sesiones de clase fueron videograbadas (250 minutos), transcritas, segmentadas y codificadas con las categorías heurísticas de Sam Wineburg. Resultados. La categoría enunciación de fuentes históricas fue la más usada para ofrecer credibilidad al relato o incluir secuencialidad temporal a los eventos del relato. La formulación de preguntas orientó la interacción entre maestra y estudiantes, y las preguntas de tipo reflexivo posibilitaron la enunciación de fuentes históricas en los estudiantes. Conclusión. Se reconstruyó la historia haciendo uso de fuentes secundarias, particularmente los libros de texto, a los cuales la maestra y sus estudiantes otorgaron credibilidad, sin someterlos a evaluación ni contrastación. Ni la maestra ni los estudiantes relativizaron, analizaron o interpretaron el conocimiento histórico reproducido en ellas. Esto hizo evidente que desconocían la forma correcta de tratar la evidencia histórica. Se sugiere explicar en la instrucción escolar tanto el uso de la heurística, para evaluar las fuentes históricas, como el uso de preguntas reflexivas.
\end{abstract}

Palabras clave. Aprendizaje, pensamiento, conocimiento, derechos humanos, historia.

\section{Use of Heuristics in the Evaluation of Historical Sources in the Classroom}

\begin{abstract}
Objective. This paper describes how the use of heuristics in the evaluation of evidence facilitated the construction of knowledge about the history of human rights in a Social Science class. Method. This qualitative study used the discourse analysis method to analyze how a social science teacher and her eighth-grade students $(n=$ 32) evaluated historical evidence. Five class sessions were recorded (250 minutes), transcribed, segmented and coded according to the categories of heuristics proposed by Sam Wineburg. Results. The enunciation

\footnotetext{
Este estudio se deriva del proyecto Explicación histórica y formas de enseñar la historia, financiado por la Universidad Pedagógica y Tecnológica de Colombia, código SGI 1596, del grupo de investigación Desarrollo humano, Cognición y Educación.

2 Magíster en Educación de la Universidad Pedagógica y Tecnológica de Colombia. Correo de correspondencia: virgelina.castellanos@uptc.edu.co

3 Doctora en Psicología de la Universidad de los Andes (Colombia).
} 
of the source categories was the most used by the teacher to provide credibility to the story or to include temporal sequences in the story. The formulation of questions guided the interaction between the teacher and their students, and reflective questions allowed a greater enunciation of historical sources from the students. Conclusion. History was reconstructed using secondary sources, particularly textbooks, to which the teacher and her students granted credibility, without subjecting them to evaluation or contrast. Neither the teacher nor the students relativized, analyzed or interpreted the historical knowledge reproduced in them. This made it clear that they were unaware of the correct way to deal with historical evidence. In the future, this study suggests explaining both the use of heuristics to evaluate the historical sources and the use of reflexive questions in the school.

Keywords. Learning, thinking, knowledge, human rights, history.

\section{Uso heurístico na avaliação das fontes históricas na sala de aula}

\section{Resumo}

Escopo. Descrever como o uso de heurísticos na avaliação de evidencia histórica possibilita a construção do conhecimento sobre a história dos direitos humanos em uma aula de Ciências Sociais. Metodologia. Estudo qualitativo, como método de análise do discurso, para analisar como a maestra e os estudantes de $8^{\circ}$ grau $(\mathrm{n}=32)$ usam e avaliam a evidencia histórica. Cinco sessões de aula foram vídeo-gravadas (150 minutos), transcritas, segmentadas e codificadas com as categorias heurísticas de San Wineburg. Resultados. A categoria enunciação de fontes históricas foi a mais usada para oferecer credibilidade aos relatos ou incluir sequencialidade temporal aos eventos do relato. A formulação de perguntas orientou a interação entre maestra e estudantes, e as perguntas de tipo reflexivo possibilitaram a enunciação de fontes históricas nos estudantes. Conclusão. Foi reconstruída a histórica fazendo uso de fontes secundárias, particularmente de livros de texto, a os quais a maestra e seus estudantes outorgaram credibilidade, sem ser sujeitos a avaliação ou contrastação. Nem a maestra nem os estudantes relativizaram, analisaram ou interpretaram o conhecimento histórico reproduzido em elas. É sugerido explicar na instruçao escolar tanto o uso da heurística, para avaliar fontes históricas, como o uso de perguntas reflexivas.

Palavras-chave. Aprendizagem, pensamento, conhecimento, direitos humanos, história.

\section{Introducción}

La política pública escolar colombiana (Ministerio de Educación Nacional [MEN], 2004) y, en consecuencia, los currículos escolares, enfatizan sobre el desarrollo de competencias básicas conceptuales y procedimentales para construir el conocimiento, tal como lo harían los historiadores, sociólogos o geógrafos. Lo esperable es que los estudiantes logren analizar, comprender e interpretar este conocimiento de forma autónoma para resolver los problemas de sus asignaturas o su vida cotidiana (MEN, 2004). En esta línea, desde la política pública, lo que se espera es que los estudiantes desarrollen la capacidad cognitiva para interpretar el mundo actual y gestionar el porvenir de la sociedad de una forma contextualizada y crítica.

Sin embargo, algunas investigaciones revelan que existen sesgos en los estudiantes para comprender el funcionamiento de los procesos sociales del pasado y establecer las relaciones de estos con el presente, tales como construir saltos temporales en las secuencias de eventos históricos, personalizar las estructuras sociales, centrar en el protagonista del relato el impulso que mueve los cambios y las transformaciones históricas (Drie y Boxtel, 2008; Navarro-Roldán y Castellanos-Páez, 2017; Navarro-Roldán y Reyes, 2016).

En su investigación, Wineburg (2001) encontró que estudiantes de secundaria y universitarios 
comprenden la evidencia como información directa y objetiva sobre el pasado, y la usan para construir sus explicaciones sin analizar su validez y veracidad. Esto quiere decir que, por lo general, los estudiantes no reconocen la necesidad de evaluar e interpretar las fuentes históricas, ni tienen en cuenta que el uso de las fuentes difiere de un relato a otro. Es posible que esto se atribuya a que en el proceso de enseñanza-aprendizaje en el aula de clase no se ofrecen los recursos y procedimientos suficientes para reconocer la importancia y las formas de usar cierto tipo de procedimientos que median la construcción del conocimiento.

En esta línea, evaluar la evidencia que soporta la construcción de conocimiento en el aula se relaciona con la calidad de la alfabetización histórica (Sáiz, 2013; Santisteban, 2010; Wineburg, 2001). Un ejemplo de esto es cuando el maestro explica el tema basándose en el uso de una sola fuente, la cual podría ser el texto escolar, a la que le otorga absoluta credibilidad y los estudiantes no tienen acceso a otros recursos, experiencias o evidencias. En consecuencia, ellos representan el pasado de la sociedad con una versión inequívoca, la cual no siempre incluye todos los aspectos, actores y relaciones causales necesarias (Ashby, 2004; Barton, 2001a, b; Barton y McCully, 2005; Britt y Aglinskas, 2002). En otras palabras, si el maestro no explica a sus estudiantes los procedimientos de indagación de fuentes disciplinares para reconstruir la ocurrencia del fenómeno a partir de ellas (p. ej.: tender líneas temporales entre la producción de las fuentes y el contexto en que fueron construidas, establecer inferencias acerca de lo que analizan las fuentes, comparar las similitudes y diferencias entre diversas fuentes que desarrollan un mismo evento), los estudiantes usarán sus teorías intuitivas para evaluar las fuentes que tienen a su disposición en el aula de clase.

Adicionalmente, la construcción sesgada del estudiante puede obtener el reconocimiento y legitimización en su contexto de aula, como lo sería una calificación sobresaliente, asentir a lo que el estudiante opina en clase o basarse solo en el texto escolar guía. Esto se daría en la medida en que se usan los recursos validados por el maestro para construir el conocimiento, a pesar de que este no use las formas disciplinares, como, por ejemplo, buena nota en el examen o reconocimiento social ante una participación en clase.
A partir de los hallazgos de la literatura, se pretende indagar si a los estudiantes en el aula de clase se les posibilitan los recursos necesarios para identificar y usar un set de herramientas o procedimientos para construir un conocimiento basado en la validez, la fiabilidad, la autenticidad y la utilidad de las fuentes de información, con el fin de evitar la construcción de sesgos en su conocimiento. Por ello, las preguntas que orientaron esta investigación fueron: ¿Cuáles son los heurísticos usados para evaluar la evidencia histórica en la construcción del conocimiento? y ¿cuáles son los recursos metodológicos usados en la interacción social en el aula que posibilitan o restringen el uso de heurísticos?

El concepto evidencia se refiere a las fuentes para construir cualquier tipo de conocimiento. En las ciencias sociales se usan fuentes primarias y secundarias -consideradas más disciplinares-, que deben articularse con las fuentes de la memoria colectiva-consideradas más populares-(Plá, 2005). Las primarias son fuentes documentales o materiales de primera mano relativas al fenómeno estudiado, como lo son las actas, la Constitución Política, la Declaración de Derechos Humanos, fuentes materiales, patrimoniales, objetos, imágenes, fotografías, pinturas. Las fuentes secundarias, por su parte, son una interpretación histórica construida a partir de las fuentes primarias en un tiempo diferente a la ocurrencia del evento pasado, tales como textos escolares, manuales, cartillas, un libro, un documental. Finalmente, las fuentes de la memoria colectiva son informales, no disciplinares, por ejemplo, un programa de televisión o narrativas orales en la familia.

Por otra parte, el concepto heurístico, siguiendo a Wineburg (2001, 1991a) y Grossman, Wineburg y Woolworth (2001), se refiere al uso de procesos cognitivos, razonamiento inductivo, o procedimientos, contrastar o discriminar, para reducir la complejidad de un problema, estimar una conclusión, explicar un evento, buscar una solución, entre otras. En sus estudios, Wineburg (1991b, 2001, 2004) encontró que los historiadores, para lograr una comprensión de los textos históricos, se ponen en el lugar del autor del libro y tratan de comprender por qué este plantea lo que escribe, o cuestionan la veracidad de la información de una fuente histórica, a partir de lo planteado en otra fuente, o analizan la secuenciación temporal de las 
imágenes de acuerdo con la información de otra fuente histórica, entre otros procedimientos.

Para Wineburg (2001), los heurísticos son habilidades susceptibles de ser desarrolladas en el aula de clase, específicamente en los procesos de alfabetización histórica. El autor encontró que los historiadores expertos usan tres heurísticos para evaluar la evidencia histórica: (a) corroboración (capacidad para comparar diferentes tipos de documentos); (b) documentación (habilidad para identificar cuál es la fuente y quién la escribió); y (c) contextualización (capacidad para reconocer cuándo y dónde tuvieron lugar los hechos reconstruidos en las fuentes).

En el presente estudio se indagó sobre las fuentes históricas usadas en una clase de Ciencias Sociales, para construir el conocimiento sobre la historia de los derechos humanos, y los heurísticos usados para tratar estas fuentes o evidencia. El dominio de los derechos humanos resulta ser un tema de vital relevancia histórica, educativa y política, para comprender el proceso de desenvolvimiento $y$ desarrollo de los seres humanos en una sociedad.

\section{Método}

\section{Diseño}

En esta investigación cualitativa se usó el método de análisis del discurso. La información recolectada fue el discurso en los procesos de interacción verbal entre la maestra y sus estudiantes (Denzin y Linconl, 2011). Cualitativamente, se identificaron las fuentes históricas y su uso. Cuantitativamente, se analizó y contrastó la presencia o ausencia de fuentes y heurísticos usados por la maestra y sus estudiantes.

\section{Participantes}

Se seleccionó de forma intencional una clase de $8^{\circ}$ grado de Ciencias Sociales en una institución educativa privada de la ciudad de Tunja (Colombia), no confesional, de nivel socioeconómico medio, en la cual interactuaron estudiantes $(n=32)$ y una maestra $(n=1)$. La edad de los estudiantes, 18 mujeres y 14 hombres, osciló entre 12.4 y 14.8 años $(M=13.7, D E=0.7)$. La maestra es licenciada en Psicopedagogía, especializada en enseñanza de las Ciencias Sociales y cuenta con 20 años de experiencia en la enseñanza de esta área.

\section{Procedimiento}

Para la recolección de los discursos, en video, se grabaron cinco sesiones de las clases de Ciencias Sociales (250 minutos), que fueron transcritas y segmentadas por línea (1236 líneas en total). El número de clases correspondió al tiempo en que la maestra desarrolló el tema, desde el inicio hasta el final. El tema abordado en el aula fue seleccionado de forma intencional, ya que trataba elementos sociales vigentes en los escenarios escolares colombianos, como lo son reivindicación social de los ciudadanos, derecho a la vida, derecho a la educación, participación social y ciudadanía, los cuales se desarrollan en el currículo obligatorio de $8^{\circ}$ grado (MEN, 2004).

Se codificó la ausencia o presencia del uso de heurísticos, documentación, corroboración y contextualización, sugeridos en el modelo de Wineburg (2001). A este modelo se agregó la categoría emergente de los datos, denominada enunciación de fuentes históricas, dado que los actores en el aula usaron de forma recurrente esta categoría en el discurso (tabla 1). Adicionalmente, se realizó un análisis descriptivo de las distribuciones encontradas a través del SPSS, versión 22. Para identificar el nivel de concordancia entre las valoraciones emitidas para cada una de las categorías analizadas, la primera investigadora codificó el $100 \%$ de las transcripciones de las entrevistas y los textos escolares, y la segunda codificó el 50\% del material, de forma independiente. El análisis de acuerdo intraclase encontrado arrojó un índice de concordancia fuerte (Kappa $\mathrm{CCl}=0.87$ ). Los desacuerdos fueron resueltos por análisis y consenso entre ambas investigadoras. 
Tabla 1

Heurísticos utilizados para evaluar la evidencia proveniente de distintas fuentes

\begin{tabular}{|c|c|c|}
\hline Categoría & Descripción & Ejemplo ${ }^{(1)}$ \\
\hline $\begin{array}{l}\text { Enunciación de } \\
\text { fuentes históricas }\end{array}$ & $\begin{array}{l}\text { Las fuentes se enuncian para } \\
\text { apoyar la explicación y se asumen } \\
\text { como evidencia inequívoca e } \\
\text { incuestionable. }\end{array}$ & $\begin{array}{l}\text { En la antigua Mesopotamia, el Código de } \\
\text { Hammurabi promulgó el primer conjunto de leyes } \\
\text { de la humanidad, y desde entonces usamos la ley } \\
\text { del Talión "ojo por ojo, diente por diente". }\end{array}$ \\
\hline Documentación & $\begin{array}{l}\text { Se describe cuál es la fuente usada } \\
\text { y quién la escribió, se identifica el } \\
\text { autor, el tiempo y el espacio donde se } \\
\text { escribió el documento. Alerta sobre } \\
\text { el tipo de texto (periodístico, novela, } \\
\text { ley, carta). }\end{array}$ & $\begin{array}{l}\text { En la antigua Mesopotamia, el Código de } \\
\text { Hammurabi fue promulgado por el rey de Babilonia, } \\
\text { Hammurabi, el cual permitió homogeneizar } \\
\text { jurídicamente y controlar el reino. El rey pensó que } \\
\text { las leyes de su territorio tenían que escribirse para } \\
\text { complacer a sus dioses. El Código se escribió en } \\
\text { bloque de basalto ( } 2.50 \mathrm{~m} \text { x } 1.90 \mathrm{~m}) \text { y fue colocado } \\
\text { en el templo de Sippar; otros similares se ubicaron } \\
\text { en diferentes lugares del reino. Durante las guerras, } \\
\text { el Código fue robado y trasladado a diferentes } \\
\text { países, y actualmente, se encuentra en el Museo del } \\
\text { Louvre, en París. }\end{array}$ \\
\hline Corroboración & $\begin{array}{l}\text { Se comparan diferentes tipos de } \\
\text { fuentes primarias (p. ej.: cartas, obras } \\
\text { de arte), secundarias (p. ej.: textos } \\
\text { escolares, documentales), la memoria } \\
\text { colectiva (p. ej.: mitos, leyendas, } \\
\text { relatos familiares) y la experiencia } \\
\text { propia (p. ej.: víctima de violación de } \\
\text { algún derecho). }\end{array}$ & $\begin{array}{l}\text { En la antigua Mesopotamia, el Código de } \\
\text { Hammurabi fue promulgado por el rey de Babilonia, } \\
\text { Hammurabi, el cual permitió homogeneizar } \\
\text { jurídicamente y controlar el reino. Sin embargo, si } \\
\text { analizamos este Código a la luz de la separación } \\
\text { de poderes en el Estado social de derecho, como } \\
\text { la de nuestro país, el Código no separó el derecho } \\
\text { civil del penal, así que regulaba la vida cotidiana, } \\
\text { el comercio, el trabajo, los préstamos, los divorcios, } \\
\text { los robos, y castigaba los delitos, etc. }\end{array}$ \\
\hline Contextualización & $\begin{array}{l}\text { Describe el marco temporal y las } \\
\text { condiciones históricas de otras } \\
\text { partes del mundo donde se gestaron } \\
\text { las fuentes utilizadas. El uso de } \\
\text { este heurístico permite analizar } \\
\text { qué precede y qué sigue a un } \\
\text { determinado acontecimiento, cuánto } \\
\text { dura y cuánto tiempo transcurrió } \\
\text { entre la ocurrencia y el registro del } \\
\text { observador. }\end{array}$ & $\begin{array}{l}\text { Durante } 1750 \text { a. C., en la antigua Mesopotamia, } \\
\text { el Código de Hammurabi fue promulgado por el } \\
\text { rey de Babilonia, Hammurabi, para homogeneizar } \\
\text { jurídicamente el reino y controlar las ciudades del } \\
\text { imperio babilónico. Actualmente, se encuentra en } \\
\text { el Museo de Louvre en París, y es un ejemplo de } \\
\text { las leyes más antiguas que se han encontrado. El } \\
\text { rey pretendió quitar poder a los sacerdotes, quienes } \\
\text { hasta entonces ejercían la justicia, y aumentar } \\
\text { el del soberano y debía ser desempeñada por sus } \\
\text { funcionarios. El Código fue escrito sobre piedra, para } \\
\text { indicar que eran inmodificables y parciales, y fueron } \\
\text { distribuidas por todo el imperio. Lo más conocido } \\
\text { de este Código ha sido la aplicación de la ley del } \\
\text { Talión, y la regulación de los salarios, precios, } \\
\text { jerarquización de la sociedad, responsabilidad, } \\
\text { justicia y penas. }\end{array}$ \\
\hline
\end{tabular}

Nota. Se ejemplifica a partir de los datos recolectados usando el Código Hammurabi, ya que fue una de las fuentes primarias más usadas por la maestra y los estudiantes.

Fuente: elaboración propia. 
Adicionalmente, en el proceso de codificación de los datos se identificó que, en la interacción entre la maestra y sus estudiantes, el recurso más usado por la profesora fue la formulación de diferentes tipos de preguntas para generar explicaciones en sus estudiantes. Por ello, estas preguntas fueron categorizadas y analizadas (tabla 2).

Tabla 2

Tipo de preguntas que orientan la interacción entre la maestra y los estudiantes

\begin{tabular}{|c|c|c|c|}
\hline $\begin{array}{l}\text { Tipo de } \\
\text { pregunta }\end{array}$ & Descripción & Indicadores & $\begin{array}{l}\text { Ejemplo de las } \\
\text { transcripciones }\end{array}$ \\
\hline Reflexiva & $\begin{array}{l}\text { Suscita la inquietud cognoscitiva y el } \\
\text { esfuerzo intelectual del alumnado y se } \\
\text { orienta, en gran medida, al análisis y } \\
\text { expresión del propio pensamiento en torno } \\
\text { al contenido. }\end{array}$ & $\begin{array}{l}\text { Generalmente inician } \\
\text { con ¿por qué? o ¿qué } \\
\text { pasaría si?, ¿qué } \\
\text { significa? }\end{array}$ & $\begin{array}{l}\text { ¿Por qué, si los derechos } \\
\text { son universales, se presenta } \\
\text { desigualdad e injusticia en } \\
\text { el país? }\end{array}$ \\
\hline Abierta & $\begin{array}{l}\text { Este tipo de pregunta no tiene respuesta } \\
\text { exacta o predeterminada. Por consiguiente, } \\
\text { admite varias respuestas, especialmente } \\
\text { descriptivas. }\end{array}$ & $\begin{array}{l}\text { Suelen iniciar } \\
\text { con pronombre } \\
\text { interrogativo: ¿Cómo? }\end{array}$ & $\begin{array}{l}\text { ¿Cómo podemos conocer } \\
\text { y hacer cumplir nuestros } \\
\text { derechos humanos? }\end{array}$ \\
\hline Literal & $\begin{array}{l}\text { Hace referencia a ideas, datos y conceptos } \\
\text { que aparecen directamente expresados en } \\
\text { un libro, un capítulo, un artículo o algún } \\
\text { otro documento. }\end{array}$ & $\begin{array}{l}\text { Usualmente inician } \\
\text { con ¿qué?, ¿cuándo?, } \\
\text { ¿dónde? }\end{array}$ & $\begin{array}{l}\text { ¿En qué fecha se proclamaron } \\
\text { los derechos humanos? }\end{array}$ \\
\hline Cerrada & $\begin{array}{l}\text { Promueve respuestas referidas a aspectos } \\
\text { puntuales del contenido, buscando } \\
\text { confirmación de la información. }\end{array}$ & $\begin{array}{l}\text { Usualmente se } \\
\text { contesta sí o no ante } \\
\text { la pregunta. }\end{array}$ & $\begin{array}{l}\text { ¿Los derechos humanos son } \\
\text { universales? }\end{array}$ \\
\hline Retórica & $\begin{array}{l}\text { Los profesores formulan la pregunta y, antes } \\
\text { de que los estudiantes puedan contestar, } \\
\text { integran la respuesta en su discurso como } \\
\text { si se tratara de un elemento semántico más. }\end{array}$ & $\begin{array}{l}\text { La pregunta } y \\
\text { la respuesta son } \\
\text { dadas por el mismo } \\
\text { hablante. }\end{array}$ & $\begin{array}{l}\text { ¿Los derechos serán iguales } \\
\text { para todos? Claro que son } \\
\text { iguales. }\end{array}$ \\
\hline
\end{tabular}

Nota. Los ejemplos son tomados de forma textual de las trascripciones analizadas.

Fuente: elaboración propia.

\section{Consideraciones éticas}

Esta investigación contó con el aval del Comité de Ética para la Investigación de la Dirección de Investigaciones de la Universidad Pedagógica y Tecnológica de Colombia, y en su ejecución se salvaguardaron todos los principios éticos y normas legales que rigen la investigación en Psicología en Colombia. Se contó con el consentimiento informado de la institución educativa, los padres de familia y el asentimiento de cada estudiante participante.

\section{Resultados}

\section{Heurísticos privilegiados en el contexto de interacción en el aula de clase}

En un total de presencia de uso de heurísticos en la interacción de 118 casos, se observó que tanto la maestra $(47.9 \%)$ como sus estudiantes $(40.3 \%)$ usaron con mayor frecuencia la enunciación de fuentes históricas. Además, la maestra usó con un bajo porcentaje el heurístico de contextualización (7.6\%) y de documentación (4.2\%). La corroboración no se presentó. 
En consecuencia, la maestra validó socialmente aquellas intervenciones de los estudiantes que enunciaron las fuentes históricas, sin relativizarlas, contrastarlas o contextualizarlas, y los estudiantes no reconocieron la utilidad de construir el conocimiento de una forma diferente, ya que la maestra después de la intervención de los estudiantes solía afirmar "muy bien", "exacto", "sí", "ajá", entre otras palabras o jergas que validan y legitiman el discurso del estudiante. A continuación, se presenta un análisis detallado para cada heurístico rastreado.

\section{Enunciación de fuentes históricas.}

La categoría emergente de los datos fue la que usaron con mayor frecuencia tanto la maestra como sus estudiantes. Esta categoría reveló que, para construir y reproducir el conocimiento de la historia de los derechos humanos, la maestra reconoció la importancia de basarse en diferentes tipos de fuentes históricas. Por su parte, los estudiantes lograron reconocer y usar en sus propias producciones discursivas recursos ofrecidos por el discurso de la maestra, en particular: (a) fuentes históricas primarias y secundarias, tales como el Código Hammurabi y el texto escolar; (b) fuentes para ofrecer credibilidad al relato, las cuales se constatan con las frases: "El libro dice", "yo lo leí", "yo lo vi"; y (c) fuentes para introducir eventos en las cadenas temporales de sus narraciones, como: "Primero fue el Código Hammurabi, luego los derechos humanos en Francia, luego la traducción de esos derechos en muchos países, luego se hacen leyes para que las personas puedan hacer cumplir los derechos".

El análisis del uso de la enunciación de fuentes históricas en las cinco sesiones, reveló que se usaron más fuentes históricas secundarias, textos escolares y cuadernos, y con menor frecuencia, la maestra usó fuentes provenientes de la memoria colectiva, videos, la experiencia propia (situaciones vividas en la cotidianidad) y fuentes primarias (el Código de Hammurabi).

a Maestra $\quad$ Estudiante

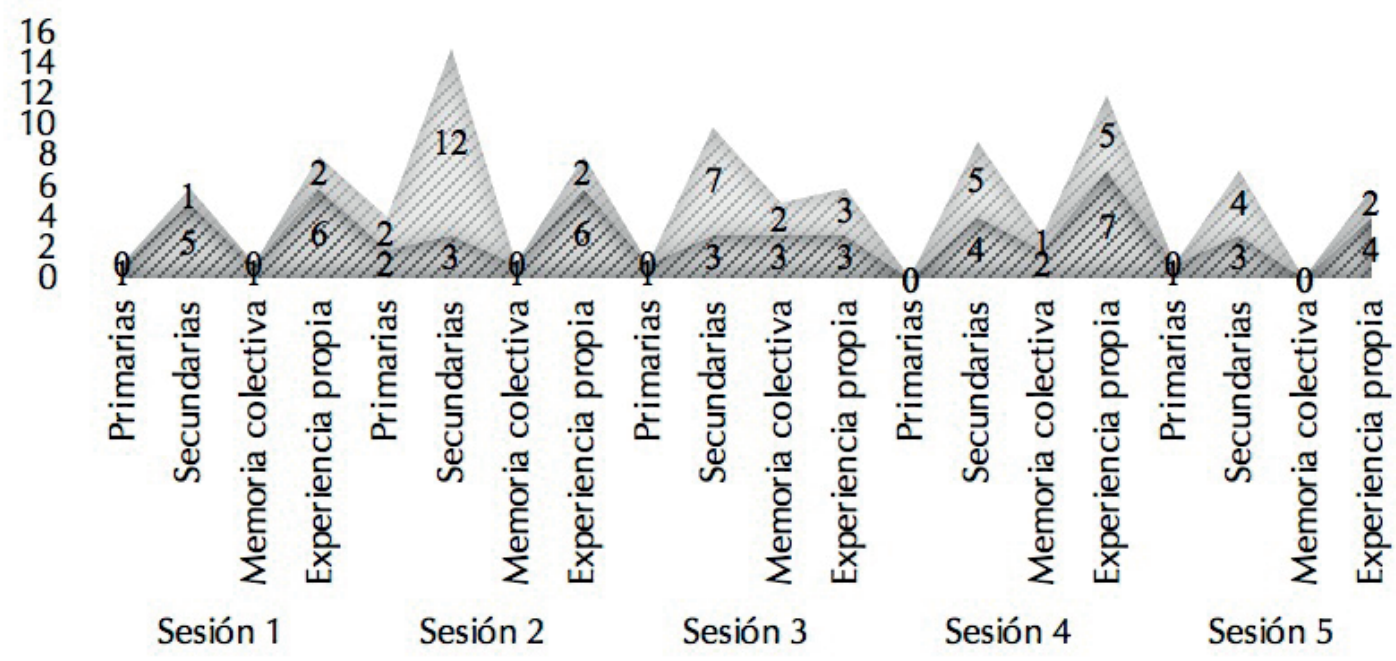

Figura 1. Distribución de frecuencias del uso de enunciación de fuentes de información historiográfica durante las cinco sesiones de clase.

Fuente: elaboración propia.

La figura 1 muestra que se usaron diferentes fuentes de información y que, por lo general, si la maestra usaba algún tipo de fuente histórica secundaria, entonces el estudiante usaba este recurso para realizar sus intervenciones. Durante las cinco clases, se fue incrementando la frecuencia del uso de la enunciación de fuentes secundarias y de la experiencia propia, para mejorar las 
intervenciones realizadas en el aula. El recuento de las distribuciones a través de la prueba exacta de Fischer reveló que se encuentran relacionados los tipos de fuentes usados por la maestra y sus estudiantes $\left(F_{(3, n=104)}=12.731 ; p=0.004\right)$.

\section{Heurístico de documentación.}

La maestra apoyó sus explicaciones especialmente en dos libros de texto de Ciencias Sociales. Usó este heurístico en las clases 1 y 2, cuando señaló como fuente primaria el Código de Hammurabi y refirió quién lo escribió, dónde y en qué fecha:

Desde que hay derechos en el mundo / el solo derecho de conseguir alimentación / derecho a tener una familia / a vivir en un lugar / esos son derechos que los tiene todo ser humano desde su nacimiento y desde que se fundó la humanidad / vamos a mirar que a partir del siglo XVII antes de Cristo se hablaba de los derechos de los seres humanos / Hammurabi habló de los derechos / si nosotros vemos el sitio, es una piedra inmensa donde están todos los derechos en el Código de Hammurabi / este es el Código de Hammurabi porque lo escribieron en piedra porque en esa época ni siquiera existía dónde escribir, entonces aparece ese código de (...) ¿este código que protege? (...) los derechos de la mujer, del niño y del esclavo. Desde esa época existía el esclavo / lo primero que hizo fue proteger los derechos en el Código de Hammurabi / ¿Dónde encontramos al rey Hammurabi? / Estudiantes en Babilonia (Relato de la maestra).

Por su parte, los estudiantes no usaron el heurístico de documentación, lo que implica que no cuestionaron ni juzgaron la validez de la fuente, considerando que la formación profesional y las motivaciones del autor pueden influir en la forma en cómo escribe y en la perspectiva que asume. Esto dificultó que los estudiantes entendieran por qué en unos documentos se narraban ciertos eventos y personajes, y en otros se omitían. Tampoco identificaron el tipo de documento que usaron (texto escolar, ley, autobiografía), ni cómo el autor Ilegó a conocer los hechos. Esto dificultó que los estudiantes tuvieran en cuenta información relevante para otorgar credibilidad y validez a los documentos que leyeron (Britt y Aglinskas, 2002).

\section{Heurístico de corroboración.}

No fue usado ni por la maestra ni por los estudiantes. La maestra no contrastó la información histórica con otras fuentes, en su lugar, extrajo información parcial de los dos textos escolares para apoyar la explicación construida, asumiendo que ambas versiones eran veraces y complementarias y que ambas aportaban en la comprensión sobre qué son los derechos humanos y cuál es su importancia en la historia. Mientras que los estudiantes se restringieron a reproducir la información que consignaron en sus cuadernos y a las consultas realizadas en los textos escolares. Esto es, asumieron que la evidencia histórica analizada era verídica y complementaria $y$, por tanto, no vieron la necesidad de contrastarla o controvertirla con otras fuentes o entre las fuentes ya usadas.

Los estudiantes tampoco usaron la corroboración para relativizar la veracidad del discurso reproducido por la maestra, así que no lograron reconocer "errores en la explicación histórica de la profesora", los cuales se denominan anacronismos históricos. Los estudiantes asumieron estos anacronismos como una verdad irrefutable, legítima y susceptible de ser reproducida en sus propias construcciones.

\section{Heurístico de contextualización.}

La maestra usó en pocas ocasiones el heurístico de la contextualización en las cinco clases, debido a que enunció lo que precede y lo que sigue a un determinado acontecimiento y señaló la temporalidad en que ocurrieron los eventos, pero no enfatizó en las condiciones sociales, económicas y políticas de la época y del lugar donde sucedieron los hechos. Tampoco señaló las transformaciones y continuidad de los fenómenos históricos, ni en qué momento y lugar fueron escritos los documentos que registraron estos hechos.

En el fragmento a continuación, de la clase 1, la maestra enunció varios eventos relacionados con la historia de la consolidación de los derechos humanos, señaló la era cronológica en que se dieron los hechos y enunció una fuente primaria, el Código de Hammurabi. Sin embargo, no profundizó en las condiciones políticas, económicas y sociales que influyeron en la aparición de los eventos, ni en qué momento y lugar fueron escritos los documentos que registraron estos hechos. 
Maestra: Desde que hay derechos en el mundo / el solo derecho de conseguir alimentación / derecho a tener una familia / a vivir en un lugar / esos son derechos que los tiene todo ser humano desde su nacimiento y desde que se fundó la humanidad / vamos a mirar cómo se empezó a hablar / vamos a mirar que a partir del siglo XVII (a.c.) se hablaba de los derechos de los seres humanos / Hammurabi habló de los derechos / si nosotros vemos el sitio, es una piedra inmensa donde están todos los derechos en el Código de Hammurabi / este es el Código de Hammurabi, lo escribieron en piedra porque en esa época ni siquiera existía dónde escribir, entonces aparece ese código de (...).

\section{Estudiantes: Hammurabi (contestan en coro).}

Maestra: Luego, en el año 5000 también antes de Cristo, se empieza a hablar de los derechos humanos en Egipto / entonces vamos a recordar nosotros que Egipto fue donde se empezó a formar la primera humanidad / fue la primera organización de los seres humanos / la primera forma de (...) sociedad / nos acordamos cuando vimos en séptimo cómo se formaron los seres humanos / mejor cómo se formaron esos grupos a través del río (...) Nilo / a través del río Nilo, la organización de seres humanos / Luego de eso también se habla de derechos cuando aparecen los griegos / los griegos organizan sus derechos humanos en el año 1500 (a.c.) / copian la línea de tiempo.

En este ejemplo, es necesario indicar que la maestra construyó un anacronismo histórico (error en la explicación histórica), al asegurar que en Egipto se inició la formación de la humanidad, lo cual induce a errores en la elaboración de las secuencias temporales de los eventos históricos. Los estudiantes, por su parte, explicaron el fenómeno de los derechos humanos a partir de una secuencia corta de eventos, no contextualizada, sin señalar las condiciones económicas, políticas y sociales de la época, ni la fecha de creación de los documentos. No tener en cuenta estos elementos cuando se usaron las fuentes, restringió la posibilidad de analizar el grado de plausibilidad y verosimilitud de las fuentes históricas usadas, incluido el discurso oral de la maestra.

\section{Interacciones en el aula de clase a través de la formulación de preguntas}

El análisis cualitativo de los procesos de interacción permitió identificar que la maestra de forma constante usó preguntas para orientar la interacción con los niños en el aula y, por ello, se procedió a codificar y clasificar las preguntas realizadas por la maestra como una categoría emergente de los datos. En el análisis se identificaron al menos cinco tipos de preguntas -reflexivas, abiertas, literales, cerradas y retóricas-, lo que permitió concluir que el incremento en la enunciación de fuentes fue posible gracias al uso de preguntas de tipo reflexivo, en la medida en que estas invitaban al estudiante a construir una explicación o justificación del por qué se empezó a hablar de derechos humanos, por qué cambiaron el funcionamiento de la sociedad, entre otros elementos. Es en estos momentos cuando los estudiantes incrementaban sus interacciones en clase, siempre apoyadas en la enunciación de algún tipo de fuente, especialmente el texto escolar y la experiencia personal. Esta tendencia fue constante durante las cinco sesiones de clase.

Al mismo tiempo, las preguntas abiertas también posibilitaron el uso de fuentes históricas, en la medida que permitieron que el estudiante describiera las situaciones leídas sobre las características de la historia de los derechos humanos en las fuentes de consulta. Sin embargo, la enunciación de fuentes de preguntas abiertas se presentó con menor frecuencia, con respecto al uso de preguntas de tipo reflexivo.

Por otro lado, las preguntas de tipo cerrado y retórico no generaron el uso de fuentes históricas, ya que requieren del estudiante respuestas cortas tipo sí o no, o de asentir o negar con la cabeza la información dada por el profesor. La tabla 4 presenta algunos ejemplos de interacciones entre la maestra y sus estudiantes, en los cuales se evidencia el tipo de preguntas y de fuentes históricas usadas por el estudiante para generar sus respuestas. 
Tabla 4

Tipos de preguntas y respuestas en los procesos de interacción en el aula de clase

\begin{tabular}{|c|c|c|}
\hline $\begin{array}{l}\text { Tipo de } \\
\text { pregunta }\end{array}$ & Pregunta de la maestra & Respuesta del estudiante \\
\hline Reflexiva & $\begin{array}{l}\text { Hablemos de la historia } \\
\text { del derecho humano a la } \\
\text { vida. } \\
\text { ¿Por qué creen que en } \\
\text { algunos lugares el aborto } \\
\text { es permitido y en otros } \\
\text { lugares es un delito? }\end{array}$ & $\begin{array}{l}\text { Los niños discuten que en Colombia es permitido en caso de } \\
\text { malformación. } \\
\text { E13: "El niño merece vivir (.) Profe, usted nos explicó que la vida es } \\
\text { un derecho (.) Eso dice en lo que hemos leído en los libros / en la } \\
\text { constitución, por ejemplo, dice que la vida es un derecho / eso es en el } \\
\text { caso de lo que el niño esté rodeado (.) en Estados Unidos hay un niño } \\
\text { sin brazos y sin piernas y salió adelante (...) ya es un hombre y tiene } \\
\text { familia //. "Mi mamá me contó / que cuando quedó embarazada de mi } \\
\text { hermano / ella tenía } 42 \text { años / y el médico le dijo /que el bebé podía } \\
\text { nacer enfermito / y que tal vez podía abortar / pero ella no quiso / así } \\
\text { como dicen los derechos humanos / mi mamá dice que ante todo está } \\
\text { el derecho a la vida / que tenía mi hermanito//". }\end{array}$ \\
\hline Abierta & $\begin{array}{l}\text { En nuestra sociedad, } \\
\text { ¿cómo se vulneran los } \\
\text { derechos humanos? }\end{array}$ & $\begin{array}{l}\text { En los noticieros siempre presentan que los niños no pueden estudiar } \\
\text { porque no tienen plata, hay gente que roba, mata. Mi mamá siempre } \\
\text { dice que tenemos que aprovechar la oportunidad de estudiar y } \\
\text { agradecer porque vivimos en un lugar que no es tan violento. }\end{array}$ \\
\hline Cerrada & $\begin{array}{l}\text { ¿En el Código } \\
\text { Hammurabi se protegían } \\
\text { los derechos y se } \\
\text { estipulaban sanciones } \\
\text { para las personas que no } \\
\text { respetaban sus normas? }\end{array}$ & ... sí \\
\hline Literal & $\begin{array}{l}\text { Estados Unidos también } \\
\text { presentó su reforma } \\
\text { hacia los derechos } \\
\text { humanos en el siglo } \\
\text { XVIII / ¿Por qué estaba } \\
\text { constituido? }\end{array}$ & Por colonias. \\
\hline Retóricas & $\begin{array}{l}\text { ¿Las reformas religiosas } \\
\text { aparecen con qué? Con } \\
\text { el Luterismo. }\end{array}$ & \\
\hline
\end{tabular}

Fuente: elaboración propia.

\section{Discusión}

En el proceso de interacción entre la maestra y los estudiantes se privilegió el desarrollo de competencias conceptuales que les permitieron a los estudiantes reconstruir la ocurrencia de los fenómenos históricos y, en ocasiones, evaluar o criticar el fenómeno a partir del contexto histórico presente. En esta línea, los objetivos de la maestra en su proceso de enseñanza giraron en torno a orientar a sus estudiantes para que ellos conceptuaran qué son los derechos humanos, reconstruyeran cuándo surgieron y cuál fue la secuencia de eventos históricos que condujeron a que estos se generaran, o explicar en qué época y contexto se dieron los eventos, y si su ocurrencia en el pasado tiene injerencia o relación con el presente, entre otros elementos. Sin embargo, la maestra realizó poco o ningún énfasis en que los estudiantes reconstruyeran la historia a partir de los 
procedimientos disciplinares, tales como el uso de heurísticos en la evaluación de las fuentes históricas, como la corroboración, la documentación y la contextualización, que disminuyeran la presencia de sesgos o anacronismos históricos, no solo en la construcción conceptual de los estudiantes, sino en el discurso de la maestra.

Los resultados también evidenciaron que en el aula se reconstruyó la historia haciendo uso de fuentes secundarias, particularmente los libros de texto, a los cuales la maestra y sus estudiantes otorgan credibilidad, sin someterlos a algún tipo de evaluación o contrastación. Regularmente, en el aula escolar, las fuentes históricas se usan para referenciar aquello en que se basan las explicaciones acerca de la historia de los derechos humanos, pero ni la maestra ni los estudiantes relativizan, analizan o interpretan el conocimiento histórico reproducido en ellas. Por ello, se entiende que tanto la maestra como los estudiantes desconocen la forma correcta de tratar la evidencia histórica (Hynd, Holschuh y Hubbard, 2004). Hallazgos similares fueron reportados por Ashby (2004), quien encontró que las fuentes históricas en la escuela se utilizan como recursos que proporcionan información directa, que no es necesario cuestionar para construir el propio conocimiento.

La manera de construir el conocimiento en el aula favoreció que los estudiantes construyeran conceptos históricos particulares, por ejemplo, secuencialidad temporal, causalidad en la ocurrencia del evento, relación entre el pasado y el presente, y que generaran explicaciones basadas en la evidencia histórica. A pesar de esto, al mismo tiempo, restringió que los estudiantes desarrollaran las competencias para explicar los fenómenos históricos a partir de la evaluación de las fuentes históricas, las cuales sirven de evidencia a priori para reconstruir la historia de los derechos humanos como una verdad relativizada a partir de diferentes fuentes (Sáiz, 2013, 2014).

De ahí que, cuando la maestra usó recursos no disciplinares para seleccionar y evaluar las fuentes, como lo fueron replicar con absoluta credibilidad el texto escolar u observar un documental sin contrastarlo con las narraciones reproducidas en los textos, se restringió la comprensión de la historia de los derechos humanos porque se aceptaron las versiones consultadas como verdades inequívocas. Esto ocurre, a pesar de que la literatura revela que el uso de heurísticos para evaluar la evidencia resulta ser un elemento clave en la construcción de conocimiento y en la formación de competencias de pensamiento histórico, tales como entendimiento de las distintas formas de representar el pasado, construcción de explicaciones basadas en la evidencia histórica, comprensión de los fenómenos históricos a partir del análisis de la evidencia y del uso de procedimientos expertos en la ciencia histórica (Santisteban, 2010; Voss y Wiley, 2006).

No obstante, a lo largo de las sesiones de clase se observó que, durante los procesos de interacción, el tipo de instrucción usada por la maestra posibilitó que los estudiantes explicaran los derechos humanos a partir de la enunciación de fuentes históricas. Cuando ella usó preguntas reflexivas como "¿qué significa ser libre en nuestro país y en nuestro colegio?"; " ¿por qué en la sociedad fue necesario escribir los derechos humanos?"; y preguntas abiertas, "¿cuáles son los principios que soportan el derecho a la vida?", “¿cuáles son los mecanismos que podemos utilizar para defender nuestros derechos?", generalmente, los estudiantes se remitían a las fuentes históricas que usaron en clase refiriendo "en el libro decía que...", "así como la profe dijo que...". Incluso, en algunas interacciones entre la maestra y los estudiantes, cuando ella realizó preguntas de tipo reflexivo, ellos articularon sus conocimientos sobre la historia de los derechos humanos con la experiencia propia, tales como anécdotas escolares o familiares.

En contraste, cuando la maestra usó preguntas cerradas y retóricas no se favoreció la construcción de explicaciones apoyadas en fuentes históricas. En estos casos, los estudiantes se limitaron a asentir con un sí o con un movimiento de la cabeza aquello que la maestra preguntaba. Esto indica que las preguntas de tipo reflexivo se convierten en un recurso metodológico importante, porque generan un desafío cognitivo en el estudiante y le permiten el avance en el desarrollo de competencias de pensamiento histórico. De forma tal que, si la maestra articulara el uso de preguntas de tipo reflexivo con el uso de heurísticos, se esperaría que al estudiante le quedara claro que los conocimientos disciplinares aprendidos desde distintas fuentes, primarias o secundarias, pueden y deben relativizarse a la luz de otras fuentes, por ejemplo, experiencia propia y memoria colectiva; en consecuencia, lograría una comprensión del fenómeno mucho más sofisticada. 
En otras palabras, se resalta que el uso de preguntas de tipo reflexivo es un recurso metodológico relevante para facilitar la construcción de explicaciones basadas en la enunciación de fuentes históricas. Sin embargo, otros recursos metodológicos pueden ser incentivados en el aula para desarrollar procedimientos que incrementen el desafío cognitivo y el desarrollo de habilidades para pensar históricamente. Algunos recursos susceptibles de ser incentivados pueden ser desconfiar de la credibilidad y la veracidad de las fuentes históricas usadas en el aula, como una estrategia para movilizar recursos cognitivos que Ileven a usar la contrastación; la contextualización y la documentación de las fuentes para construir representaciones del pasado de la sociedad de formas cada vez más sofisticadas a nivel disciplinar, con el fin de reducir la posibilidad de sesgos o anacronismos históricos (Ashby, 2004; Drie y Boxtel, 2008; Sáiz, 2013, 2014; Vázquez y Mugueta, 2012; Wineburg, 2001).

Otras estrategias pueden ser llevar a cabo coloquios con preguntas reflexivas que conduzcan al estudiante a relacionar el pasado con el presente, elaborar cuadros comparativos sobre diferentes perspectivas de un mismo fenómeno histórico y ponerse en contacto directo con el pasado a través de revisiones documentales en archivos históricos o visitas a museos. Además, realizar ejercicios de relativización de los relatos históricos, comparando la fuente primaria con entrevistas a familiares, a fin de recuperar la memoria colectiva.

Todas estas estrategias aportarían en el proceso de mediación para facilitar que el estudiante desarrolle diversas competencias (Barton y Levstik, 2004) y se aproxime a la reconstrucción de la historia desde modelos expertos (Carretero y López, 2009; Voss y Wiley, 2006; Wineburg, 2001). Así, la enseñanza de la historia iría en la misma línea a lo propuesto en los estándares curriculares colombianos que buscan que el estudiante de Ciencias Sociales se aproxime al conocimiento como científico social, de la misma forma como proceden los historiadores en la reconstrucción de la historia, la comprensión y la construcción de un mejor entorno social (MEN, 2004).

Se resalta que la evaluación de fuentes en la enseñanza de las Ciencias Sociales, es un recurso que permite que los estudiantes simulen la labor del experto y con ello aprendan a formular hipótesis, argumentar y construir explicaciones históricas sustentadas en el análisis de la evidencia histórica. Esto lleva a que los estudiantes asuman una mirada crítica y constructiva en el aprendizaje de la historia en los escenarios escolares (Hoodless, 2004; Pagés y Santisteban, 2010; Prieto, Gómez y Miralles, 2013; Sáiz, 2013, 2014).

Cabe señalar que este estudio arrojó información valiosa sobre el uso de los heurísticos en la evaluación de fuentes históricas en un contexto de aula y aportó elementos que enriquecen la enseñanza de las Ciencias Sociales. Sin embargo, los hallazgos deben analizarse a la luz de las limitaciones de la investigación. En primer lugar, aunque los hallazgos de este estudio se orientan en la misma línea de estudios previos (Ashby, 2004; Wineburg, 2001), sus resultados no son generalizables a diferentes contextos de enseñanza. Recabar hallazgos concluyentes requiere aunar esfuerzos para ampliar la investigación a otro tipo de escuelas, como públicas y privadas, rurales y urbanas, y grados escolares.

En segundo lugar, sería útil proponer estudios que establezcan un análisis con relación a otro tipo de variables y competencias disciplinares. Por ejemplo, analizar cómo el uso de las fuentes históricas se relaciona con el nivel de formación docente, profesión y años de experiencia, entre otras variables; además, cómo los maestros favorecen en las aulas el entendimiento de la causalidad, la conciencia histórico-temporal y la empatía histórica.

En consecuencia, es pertinente continuar indagando sobre las prácticas educativas al enseñar las ciencias sociales y la historia, con la finalidad de generar reflexiones en torno a los elementos que posibilitan o restringen la formación de competencias históricas. Asimismo, los resultados permiten sugerir el diseño, implementación y evaluación de herramientas pedagógicas que incluyan en la formación de maestros el aprendizaje de la historia escolar desde modelos expertos que acerquen la labor del estudiante a la del historiador.

\section{Referencias}

Ashby, R. (2004). Developing a Concept of Historical Evidence: Students' ideas About Testing Singular Factual Claims. International Journal 
of Historical Learning, Teaching and Research, 4(2), 151-170. Recuperado de https://www. history.org.uk/secondary/resource/4858/theinternational-journal-volume-4-number-2

Barton, K. C. (2001a). Primary Children's Understanding of the Role of Historical Evidence: Comparisons between the United States and Northern Ireland. International Journal of Historical Learning, 1(2), 13-19. doi: 10.18546/HERJ.01.2.03

Barton, K. C. (2001b). A Sociocultural Perspective on Children's Understanding of Historical Change: Comparative Findings from Northern Ireland and the United States. American Educational Research Journal, 38(4), 881-913. https://doi. org/10.3102/00028312038004881

Barton, K. C., \& Levstik, L. S. (2004). Teaching History for the Common Good. Mahwah, NJ: Erlbaum.

Barton, K. C., \& McCully, A. W. (2005). History, Identity, and the School Curriculum in Northern Ireland: An Empirical Study of Secondary Students' ideas and Perspectives. Journal of Curriculum Studies, 37(1), 85-116. doi: 10.1080/0022027032000266070

Britt, M. A., \& Aglinskas, C. (2002). Improving Students' Ability to Identify and Use Source Information. Cognition and Instruction, 20(4), 485-522. Recuperado de https://www.niu.edu/ britt/recent_papers/pdfs/Britt_Aglinski_2002. pdf

Carretero, M. y López, C. (2009). Estudios cognitivos sobre el conocimiento histórico: aportaciones para la enseñanza y alfabetización histórica. Enseñanza de las Ciencias Sociales, 8, 79-93. Recuperado de https://www.raco.cat/index. php/EnsenanzaCS/article/view/184396

Denzin, N. K., \& Lincoln, Y. S. (Eds.). (2011). The Sage Handbook of Qualitative Research. Thousand Oaks, CA: Sage Publications.

Drie, J. van, \& Boxtel, C. van (2008). Historical Reasoning: Towards a Framework for Analyzing Students' Reasoning about the Past. Educational Psychology Review, 20(2), 87-110. doi: 10.1007/s10648-007-9056-1

Grossman, P., Wineburg, S., \& Woolworth, S. (2001). Toward a Theory of Teacher Community.
The Teachers College Record, 103(6), 9421012. Recuperado de http://www.tcrecord.org/ Content.asp?ContentID=10833

Hoodless, P. (2004). Spotting the Adult Agendas: Investigating Children's Historical Awareness Using Stories Written for Children in the Past. International Journal of Historical Learning, Teaching and Research, 4(2), 66-75. doi: 10.18546/HERJ.04.2.08

Hynd, C., Holschuh, J. P., \& Hubbard, B. P. (2004). Thinking Like a Historian: College Students' reading of Multiple Historical Documents. Journal of Literacy Research, 36(2), 141-176. doi: 10.1207/s15548430jlr3602_2

Ministerio de Educación Nacional [MEN]. (2004). Formar en Ciencias: iel desafío! Lo que necesitamos saber y saber hacer. Serie Guía No. 7. Estándares Básicos de Competencias en Ciencias Naturales y Ciencias Sociales. Colombia: Ascofade. Recuperado de http://www.eduteka.org/pdfdir/ MENEstandaresCienciasSociales2004.pdf

Navarro-Roldán, C. P. y Castellano-Páez, V. (2017). Cambio conceptual en el aprendizaje escolar de la Historia. Revista CES de Psicología, 10(2), 1-16. http://dx.doi.org/10.21615/cesp.10.2.1

Navarro-Roldán, C. P. y Reyes, P. A. (2016). Cambios cognitivos en la forma de explicar la historia en estudiantes de quinto grado. Pensamiento Psicológico, 14(2), 63-75. doi:10.11144/ Javerianacali.PPSI14-2.ccfe

Pagés, J. y Santisteban, A. (2010). La enseñanza y aprendizaje del tiempo histórico en la Educación Primaria. Cad. Cedes, Campinas, 30(82), 281-309. Recuperado de http://www. scielo.br/pdf/ccedes/v30n82/02.pdf

Plá, S. (2005). Aprender a pensar históricamente: la escritura de la historia en el bachillerato. México DF: Plaza y Valdés.

Prieto, J. A., Gómez, C. J. y Miralles, P. (2013). El uso de fuentes primarias en el aula y el desarrollo del pensamiento histórico y social: una experiencia en Bachillerato. Clío. History and History Teaching, 39, 1-14. Recuperado de http://clio.rediris.es/n39/articulos/historia social/PrietoGomezMiralles.pdf 
Sáiz, J. (2013). Alfabetización histórica y competencias básicas en libros de texto de historia y en aprendizaje de los estudiantes. Didáctica de las Ciencias Experimentales $y$ Sociales, 27(4), 43-66. doi:10.7203/ DCES.27.2648

Sáiz, J. (2014). Fuentes históricas y libros de texto en secundaria: una oportunidad perdida para enseñar competencias de pensamiento histórico. Ensayos, 29(1), 83-99. Recuperado de https://revista.uclm.es/index.php/ensayos/ article/view/503/458

Santisteban, A. (2010). La formación de competencias de pensamiento histórico. Clío \& Asociados (14), 34-56. Recuperado de http:// www.memoria.fahce.unlp.edu.ar/art_revistas/ pr.4019/pr.4019

Vázquez, A. y Mugueta, I. (2012). Utilización de las fuentes primarias para la enseñanza de la historia (Trabajo fin de Máster en Formación del Profesorado en Secundaria). Universidad de Navarra, España.

Voss, J. F., \& Wiley, J. (2006). Expertise in History. En K. A. Ericsson, R. R. Hoffman, A. Kozbelt, \& A.
M. Williams (Eds.), The Cambridge Handbook of Expertise and Expert Performance (pp. 569584). Cambridge, England, UK: Cambridge University Press.

Wineburg, S. (1991a). Historical Problem Solving: A Study of the Cognitive Processes Used in the Evaluation of Documentary and Pictorial Evidence. Journal of Educational Psychology, 83(1), 73-87. Recuperado de http://citeseerx. ist.psu.edu/viewdoc/download; jsessionid=DC 02C8C05F46E76003C3AE624D4F89CF?doi= 10.1.1.1030.5326\&rep=rep1 \&type $=$ pdf

Wineburg, S. (1991b). On the Reading of Historical Texts: Notes on the Breach between School and Academy. American Educational Research Journal, 28(3), 495-519. doi: 10.3102/00028312028003495

Wineburg, S. (2001). Historical Thinking and Other Unnatural Acts. Charting the Future of Teaching the Past. Philadelphia: Temple University Press.

Wineburg, S. (2004). Crazy for history. The Journal of American History, 90(4), 1401-1414. doi: $10.2307 / 3660360$

Para citar este artículo / To cite this article / Para citar este artigo: Castellanos-Páez, V. y Navarro-Roldán, C.P. (2020). Uso de heurísticos en la evaluación de las fuentes históricas en el aula de clase. Pensamiento Psicológico, 18(1), 43-56. doi:10.11144/Javerianacali. PPSI18-1.uhef 